\title{
Intraoperative and postoperative complications of gynecological laparoscopic interventions: incidence and risk factors
}

\author{
A. C. Kaya ${ }^{1}$ (1) M. P. Radosa ${ }^{2}$ - J. S. M. Zimmermann ${ }^{1}$ - L. Stotz ${ }^{1}$ - S. Findeklee ${ }^{1}$ - A. Hamza ${ }^{1,3}$ - P. Sklavounos ${ }^{1}$. \\ F. Z. Takacs ${ }^{1}$ - G. Wagenpfeil ${ }^{4}$. C. G. Radosa ${ }^{5}$. E. F. Solomayer ${ }^{1}$. J. C. Radosa ${ }^{1}$
}

Received: 25 April 2021 / Accepted: 14 August 2021 / Published online: 21 August 2021

(c) The Author(s) 2021

\begin{abstract}
Purpose The aims of this study were to determine the incidence of intraoperative and postoperative complications of laparoscopic gynecological interventions and to identify risk factors for such complications.

Methods All patients who underwent laparoscopic interventions from September 2013 to September 2017 at the Department of Gynecology, Obstetrics and Reproductive Medicine, Saarland University Hospital were identified retrospectively using a prospectively compiled clinical database. Binary logistic regression analysis was used to identify independent risk factors for intra- and postoperative complications.

Results Data from 3351 patients were included in the final analysis. Overall, $188(5.6 \%)$ intraoperative and $219(6.5 \%)$ postoperative complications were detected. On multivariate analysis, age [odds ratio (OR), 1.03; 95\% confidence interval (CI) 1.01-1.04], surgery duration (OR, 1.02; 95\% CI 1.02-1.03), carbon dioxide use (OR, 0.99; 95\% CI 0.99-1.00), and surgical indication (all $p \leq 0.01$ ) were independent risk factors for intraoperative and duration of surgery (OR, 1.01; 95\% CI $1.01-1.02 ; p \leq 0.01)$, carbon dioxide use (OR, 0.99; 95\% CI 0.99-1.00; $p \leq 0.01)$, hemoglobin drop (OR, $1.41 ; 95 \%$ CI $1.21-1.65 ; p \leq 0.01)$, and ASA status $(p=0.04)$ for postoperative complications.

Conclusion In this large retrospective analysis with a generally low incidence of complications (5.6\% intraoperative and $6.5 \%$ postoperative complications), a representative risk collective was identified: Patients aged $>38$ years, surgery duration $>99$ min, benign or malignant adnex findings were at higher risk for intraoperative and patients with surgery duration $>94 \mathrm{~min}$, hemoglobin drop $>2 \mathrm{~g} / \mathrm{dl}$ and ASA status III at higher risk for postoperative complications.
\end{abstract}

Keywords Laparoscopy $\cdot$ Gynecology $\cdot$ Postoperative complication $\cdot$ Minimally invasive surgery $\cdot$ Hysterectomy

\section{Introduction}

A. C. Kaya

a.kaya3@gmx.de

1 Department of Gynecology, Obstetrics and Reproductive Medicine, Saarland University Hospital, Homburg/Saar, Germany

2 Department of Gynecology and Obstetrics, Klinikum Bremen-Nord, Bremen, Germany

3 Department of Gynecology and Obstetrics, Kantonsspital Baden, Baden, Switzerland

4 Institute of Medical Biometry, Epidemiology \& Medical Informatics, Saarland University Hospital, Homburg/Saar, Germany

5 Department of Diagnostic and Interventional Radiology, University Hospital Carl Gustav Carus, TU, Dresden, Germany
Minimally invasive techniques have replaced a plethora of open surgical interventions [1]. Numerous studies have shown that laparoscopy offers many advantages over open surgery, such as reduced intraoperative blood loss, postoperative pain levels, hospitalization and faster recovery times, including for obese patients, general surgeries, and the treatment of benign and malignant gynecological diseases [2-6]. Although minimally invasive techniques are the gold standards in many fields of surgery, they are intra-abdominal procedures, and complications of laparoscopic interventions are not less severe [7]. Reported overall complication rates vary between different authors, with ranges of overall complication rates between $4-41.21 \%$, possibly due to differences in study design and cohort size [8, 17-20]. In addition, a major problem in research on laparoscopic complications is the 
lack of standardized definition and recording of complications. The aim of this observational study was to determine the rates of intraoperative and postoperative complications of laparoscopic gynecological interventions, and to identify risk factors for the occurrence of these complications.

\section{Materials and methods}

\section{Study design and participants}

All patients who underwent laparoscopy at the Department for Gynecology, Obstetrics and Reproductive Medicine, Saarland University Hospital between September 2013 and September 2017 were identified retrospectively using a prospectively compiled institutional clinical database. All patients who underwent laparoscopic gynecological surgeries were included in this study. Those who underwent laparoscopically assisted vaginal hysterectomies and those for whom intervention or patient data were incomplete were excluded.

A gynecological fellow collected the study data by systemic chart review. Data on patient characteristics [age (years), height $(\mathrm{cm})$, weight $(\mathrm{kg})$, body mass index (BMI; $\left.\mathrm{kg} / \mathrm{m}^{2}\right)$ ], surgical parameters such as surgery duration ( $\mathrm{min}$ ), carbon dioxide $\left(\mathrm{CO}_{2}\right)$ use (l) (defined as amount of carbon dioxide in liter insufflated from the insertion of the Veress needle to the removal of the last trocar), hemoglobin ( $\mathrm{Hb}$ ) drop ( $\mathrm{g} / \mathrm{dl}$ ) (defined as difference between the last preoperative and the first postoperative hemoglobin control in $\mathrm{g} / \mathrm{dl}$ ), surgical indications [symptomatic uterine myoma, benign or malignant adnexal finding, cervical cancer, endometrial carcinoma, endometriosis, urogynecological indication, cervical intraepithelial neoplasia (CIN)], histological findings (benign/malignant), laparoscopic intervention difficulty according to Barakat [low-medium (levels I and II), medium high-high (levels III and IV)], American Society of Anesthesiologists (ASA) physical status, adhesiolysis and duration of postoperative hospital stay (days) were extracted [9, $10]$.

Surgical complications were recorded as intra- and postoperative. Bleeding, blood transfusion, organ injury, conversion to laparotomy, resuscitation and skin emphysema were defined as relevant intraoperative complications. Postoperative complications (all deviations from the normal postoperative course) occurring during a 6-week period after surgery were classified using the five-grade Clavien-Dindo system and as minor (levels I and II) and major (levels III to $\mathrm{V})[8,11]$.

\section{Procedures}

All laparoscopies were performed by experienced consultants. All patients received perioperative antibiotic prophylaxis with cefuroxime (single shot, 1.5 g i.v.; Fresenius Kabi, Bad Homburg, Germany) and, if necessary, metronidazole (single shot, 500 mg i.v; Fresenius Kabi, Bad Homburg, Germany) and a perioperative gastric tube and indwelling urinary catheter until mobilization on the first postoperative morning. For perioperative thromboembolism prophylaxis, low-molecular-weight heparin (enoxaparin sodium, $40 \mathrm{mg}$; Sanofi, Paris, France) was injected daily during patients' entire hospital stays [12]. For hysterectomies of benign and malignant indication, such prophylaxis was administered for 1 and 4 weeks, respectively. Laparoscopy was performed in lithotomy position using the four-port technique; specifics of the techniques used for different interventions have been described previously [12-16]. After surgery, all patients were taken to postoperative ward for 4 to 6 hours of monitoring vital signs, then moved to general ward; blood count control was performed on the first postoperative day. Before discharge from hospital, final examination with speculum insertion and transvaginal and kidney ultrasound was performed in all cases. Patients were advised to present to the emergency ward if any deviation from the normal postoperative course occurred.

\section{Statistical analysis}

All data were collected with Microsoft Excel (Excel 2014, Microsoft Corporation, Redmond, WA, USA). The statistical analysis was performed using IBM SPSS (Version 25, IBM SPSS Inc., Chicago, IL, USA). For quantitative data, the Kolmogorov-Smirnov test was used to assess the normality of distribution. As the data were not distributed normally, medians and ranges were calculated and the Mann-Whitney $U$ test was used for comparison. For categorical variables, absolute and relative frequencies were determined, and the Pearson chisquared test was used for comparison. Patient characteristics and surgical parameters were compared according to the presence or absence of intraoperative and postoperative complications. Multivariate binary logistic regression with stepwise forward and backward selection was used to identify factors predicting the occurrence of these complications. A receiver operating characteristic (ROC) curve was used to vary the discrimination thresholds for quantitative risk factors. 


\section{Results}

\section{Patient characteristics and surgical parameters}

Of 3409 patients identified from the database, 58 were excluded due to lack of information; thus, data from 3351 patients were examined in the present analysis. The patient characteristics and surgical parameters are presented in Table 1. The median patient age was 42 (range 9-95) years and the median BMI was 25.1 (range $15.6-62.5$ ) $\mathrm{kg} / \mathrm{m}^{2}$. The median duration of surgery was 82 (range 2-492) min. A median of 156 (range 7.4-2060) 1 carbon dioxide was used during surgery and a median hemoglobin drop of 1.0 (range $-4.2-8.5) \mathrm{g} / \mathrm{dl}$ occurred. The median length of hospital stay was 3 (range $0-75$ ) days. The most common indication for surgery was a benign adnexal finding $[n=900$ (26.9\%)]. Malignancy was diagnosed in 421 (12.6\%) cases, and $2930(87.4 \%)$ laparoscopies were performed due to benign findings. Most $[n=2484(74.1 \%)]$ interventions were of technical difficulty level II and most patients were classified with a Grade II ASA status [ $n=2080(62.1 \%)]$. Adhesiolysis was performed during 1950 (58.2\%) laparoscopies (Table 1).

\section{Intra- and postoperative complications}

Intraoperative complications were recorded in 188 (5.6\%) cases. Conversion to laparotomy was the most common such complication $[n=92(2.7 \%)]$, followed by organ injury $[n=75(2.2 \%)]$. Organ injuries were recorded for the intestinal tract $[n=35(1.0 \%)]$, bladder $[n=29(0.9 \%)]$, ureter $[n=3(0.1 \%)]$, blood vessel $[n=1(0.02 \%)]$, and "others" [e.g., uterus, diaphragm; $n=7(0.2 \%)$ ]. Other intraoperative complications were bleeding $[n=1$ $(0.02 \%)]$, the need for blood transfusion $[n=17(0.5 \%)]$ and resuscitation $[n=2(0.1 \%)]$, and skin emphysema $[n=1(0.02 \%)]$. Conversion to open surgery was performed due to complications in $62(1.9 \%)$ cases and suspected or confirmed malignancy in $30(0.9 \%)$ cases.

Postoperative complications were recorded in 219 (6.5\%) cases; 92 (2.7\%) were minor and 128 (3.8\%) were major. According to clinical manifestation, revision of postoperative hemorrhage and hematomas was applied most commonly $[n=41(1.2 \%)]$, followed by the antibiotic treatment of infections, hematomas, and abscesses $[n=28(0.8 \%)]$ and transfusion for anemia $[n=26(0.8 \%)]$. Twenty-three $(0.7 \%)$ patients presented at the hospital due to the post-discharge exacerbation of pain, $13(0.4 \%)$ patients had cardiopulmonary complications, $7(0.2 \%)$ patients had ileuses treated conservatively with prokinetic stimulation, and $5(0.1 \%)$ patients had ileuses requiring
Table 1 Patient characteristics and surgical parameters

Patient characteristics and surgical parameters $(n=3351)$

\begin{tabular}{|c|c|}
\hline & $\begin{array}{l}\text { Median (mini- } \\
\text { mum-maxi- } \\
\text { mum) }\end{array}$ \\
\hline Age (years) & $42(9-95)$ \\
\hline Weight (kg) & $68(33-172)$ \\
\hline Body mass index $\left(\mathrm{kg} / \mathrm{m}^{2}\right)$ & $25.1(15.6-62.5)$ \\
\hline Operative time (min) & $82(2-492)$ \\
\hline Carbon dioxide use (1) & $156(7.4-2060)$ \\
\hline Hemoglobin drop (g/dl) & $1(-4.2-8.5)$ \\
\hline \multirow[t]{2}{*}{ Length of hospital stay (days) } & $3(0-75)$ \\
\hline & $n(\%)$ \\
\hline \multicolumn{2}{|l|}{ Indication } \\
\hline Benign adnexal finding & $900(26.9 \%)$ \\
\hline Uterus myomatosus & $823(24.6 \%)$ \\
\hline Endometriosis & $537(16.0 \%)$ \\
\hline Others & $494(14.7 \%)$ \\
\hline \multicolumn{2}{|l|}{ Urogynecological } \\
\hline Indication & $162(4.8 \%)$ \\
\hline Endometrial cancer & $151(4.5 \%)$ \\
\hline Cervical cancer & $131(3.9 \%)$ \\
\hline Malignant adnexal finding & $115(3.4 \%)$ \\
\hline $\mathrm{CIN}$ & $38(1.1 \%)$ \\
\hline \multicolumn{2}{|l|}{ Malignancy } \\
\hline Yes & $421(12.6 \%)$ \\
\hline No & $2930(87.4 \%)$ \\
\hline \multicolumn{2}{|l|}{ Barakat-classification } \\
\hline Grade II & $2484(74.1 \%)$ \\
\hline Grade III & $461(13.8 \%)$ \\
\hline Grade IV & $345(10.3 \%)$ \\
\hline Grade I & $61(1.8 \%)$ \\
\hline \multicolumn{2}{|l|}{ ASA-classification } \\
\hline Grade II & $2080(62.1 \%)$ \\
\hline Grade I & $880(26.3 \%)$ \\
\hline Grade III & $386(11.5 \%)$ \\
\hline Grade IV & $5(0.1 \%)$ \\
\hline Grade V & $0(0.0 \%)$ \\
\hline Grade VI & $0(0.0 \%)$ \\
\hline \multicolumn{2}{|l|}{ Adhesiolysis } \\
\hline Yes & $1950(58.2 \%)$ \\
\hline No & $1401(41.8 \%)$ \\
\hline
\end{tabular}

CIN cervical intraepithelial neoplasia, ASA-Classification American Society of Anaesthesiologists Classification $(n=3351)$

surgery. Minimal vaginal cuff dehiscence that could be treated conservatively was documented in $3(0.1 \%)$ cases; $12(4 \%)$ cases of dehiscence requiring re-intervention occurred. Eighteen $(0.5 \%)$ patients with intestinal problems (e.g., peritonitis, anastomosis insufficiency, fistula) and four $(0.1 \%)$ patients with bladder injury, ureteral 
lesion, or vesicovaginal fistula required surgical treatment. Nerve lesions and paresthesia were registered in five $(0.1 \%)$ cases, trocar site hernia or infection were recorded in two $(0.1 \%)$ cases, lymphocele was recorded in one $(0.02 \%)$ case, and compartment syndrome was recorded in two $(0.1 \%)$ cases. Wound healing disorders were treated conservatively in two $(0.1 \%)$ cases and operatively in four $(0.1 \%)$ cases. Eight $(0.2 \%)$ abscess revisions were recorded. Three $(0.1 \%)$ patients had multiorgan dysfunction and $12(0.4 \%)$ patients experienced other types of adverse event (Table 2).

\section{Associations of surgical parameters with intraoperative complications}

The intraoperative complication rate differed significantly between patients with benign and malignant diagnoses (112 $(59.6 \%$ vs. $76(40.4 \%, p \leq 0.01)$. It further differed according to surgical indication [uterine myomas $(n=44 ;(23.4 \%$ vs. benign adnexal findings $(n=35 ;(18.6 \%$ vs. malignant adnexal findings $(n=31 ;(16.5 \%$ vs. cervical cancer $(n=17$; (9.0\% vs. endometrial cancer $((n=23 ; 12.2 \%$ vs. endometriosis $(n=16 ;(8.5 \%$ vs. urogynecological conditions $((n=6$; $3.2 \%$ vs. CIN ( $n=0,(0 \%$ vs. other conditions $((n=16,8.5 \%$, $p \leq 0.01]$. In addition, the occurrence of intraoperative complications differed according to the laparoscopy complexity level [(I: 0 (0\%) vs. II: 87 (46.3\%) vs. III: 32 (17\%) vs. IV: $69(36.7 \%), p \leq 0.01$ ], ASA status [I: $27(14.4 \%)$ vs. II: 109 (58\%) vs. III: 51 (27.1\%) vs. IV: $1(0.5 \%), p \leq 0.01]$ and BMI [underweight: $8(4.3 \%)$ vs. normalweight: $79(42.0 \%)$ vs. overweight: $38(20.2 \%)$ vs. obesity I: $32(17 \%)$ vs. obesity II: $12(6.4 \%)$ vs. obesity III: $19(10.1 \%), p \leq 0.01]$.

Relative to those without intraoperative complications, patients with such complications were significantly older [42 (range 9-95) vs. 50 (range 21-87) years, $p \leq 0.01$ ], with longer surgeries [79 (range 2-415) vs. 180 (range 24-492) min, $p \leq 0.01$ ], greater $\mathrm{CO}_{2}$ use [153 (range 7.4-2060) vs. 255 (range 7.7-1373) $1, p=0.02$ ], larger hemoglobin drops [1.0 (range - 2.2-8.5) vs. 1.5 (range $-4.2-5.7$ ) g/ $\mathrm{dl}, p \leq 0.01$ ], higher BMIs [25 (range 15.6-62.5) vs. 26.1 (range 16.3-59.5) kg/m $\mathrm{m}^{2}, p \leq 0.01$ ] and longer hospital stays [3 (range $0-72$ ) vs. 7 (range $0-75$ ) days, $p \leq 0.01$ ]. The intraoperative complication rate did not differ with regard to performance of adhesiolysis (Table 3).

\section{Associations of surgical parameters with postoperative complications}

The postoperative complication rate differed significantly between patients with benign and malignant diagnoses (161 (73.5\%) vs. $58(26.5 \%), p \leq 0.01)$, and further according to surgical indication [uterine myoma $(n=60 ; 27.4 \%)$ vs. benign adnexal findings $(n=35 ; 16 \%)$ vs. malignant adnexal
Table 2 Intra- and postoperative complications

\begin{tabular}{|c|c|}
\hline \multicolumn{2}{|l|}{ Intra- and postoperative complications $(n=3351)$} \\
\hline Intraoperative complications & Total (\%) \\
\hline Yes & $188(5.6 \%)$ \\
\hline No & $3163(94.4 \%)$ \\
\hline \multicolumn{2}{|l|}{ Type of complication } \\
\hline Conversion to laparotomy & $92(2.7 \%)$ \\
\hline Due to complication & $62(1.9 \%)$ \\
\hline Due to dignity & $30(0.9 \%)$ \\
\hline Organ injury & $75(2.2 \%)$ \\
\hline Intestine & $35(1.0 \%)$ \\
\hline Bladder & $29(0.9 \%)$ \\
\hline Ureter & $3(0.1 \%)$ \\
\hline Vessel & $1(0.02 \%)$ \\
\hline Others & $7(0.2 \%)$ \\
\hline Blood transfusion & $17(0.5 \%)$ \\
\hline Resuscitation & $2(0.1 \%)$ \\
\hline Skin emphysema & $1(0.02 \%)$ \\
\hline Bleeding & $1(0.02 \%)$ \\
\hline \multicolumn{2}{|l|}{ Postoperative complications } \\
\hline Yes & $219(6.5 \%)$ \\
\hline No & $3132(93.5 \%)$ \\
\hline \multicolumn{2}{|l|}{ Type of complication } \\
\hline CD IIIb & $90(2.7 \%)$ \\
\hline CD II & $65(1.9 \%)$ \\
\hline CD I & $27(0.8 \%)$ \\
\hline CD IVa & $24(0.7 \%)$ \\
\hline CD IIIa & $11(0.3 \%)$ \\
\hline $\mathrm{CD} \mathrm{V}$ & $3(0.1 \%)$ \\
\hline $\mathrm{CD} \mathrm{IVb}$ & $0(0.0 \%)$ \\
\hline \multicolumn{2}{|l|}{ Clinical manifestation of postoperative complications } \\
\hline Bleeding/hematoma-revision & $41(1.2 \%)$ \\
\hline Infection/antibiotics/hematoma/abscess & $28(0.8 \%)$ \\
\hline Anemia requiring transfusion & $26(0.8 \%)$ \\
\hline Pain/adhesions & $23(0.7 \%)$ \\
\hline $\begin{array}{l}\text { Intestinal injury/peritonitis/anastomosis insuffi- } \\
\text { ciency/fistula-operative therapy }\end{array}$ & $18(0.5 \%)$ \\
\hline Cardiopulmonal/thrombosis/embolism & $13(0.4 \%)$ \\
\hline Vaginal cuff dehiscence-operative therapy & $12(0.4 \%)$ \\
\hline Others & $12(0.4 \%)$ \\
\hline Abscess-operative therapy & $8(0.2 \%)$ \\
\hline Ileus-conservative therapy & $7(0.2 \%)$ \\
\hline Ileus-operative therapy & $5(0.1 \%)$ \\
\hline Nerve lesion/paresthesia & $5(0.1 \%)$ \\
\hline $\begin{array}{l}\text { Bladder injury/ureter injury/vesicovaginal fistula- } \\
\text { operative therapy }\end{array}$ & $4(0.1 \%)$ \\
\hline Wound healing disorder-operative therapy & $4(0.1 \%)$ \\
\hline Vaginal cuff dehiscence-conservative therapy & $3(0.1 \%)$ \\
\hline Multiorgan disfunction/death & $3(0.1 \%)$ \\
\hline Trocar site hernia/infection & $2(0.1 \%)$ \\
\hline Wound healing disorder-conservative therapy & $2(0.1 \%)$ \\
\hline Compartment syndrome/ limb ischemia & $2(0.1 \%)$ \\
\hline
\end{tabular}


Table 2 (continued)

Intra- and postoperative complications $(n=3351)$

\begin{tabular}{ll}
\hline Intraoperative complications & Total $(\%)$ \\
\hline Lymphocele & $1(0.02 \%)$ \\
\hline
\end{tabular}

$C D$ Clavien Dindo $(n=3351)$

findings $(n=20 ; 9.1 \%)$ vs. cervical cancer $(n=10 ; 4.6 \%)$ vs. endometrial cancer $(n=23 ; 10.5 \%)$ vs. endometriosis $(n=24 ; 11 \%)$ vs. urogynecological indications $(n=15$; $6.8 \%)$ vs. CIN $(n=2,0.9 \%)$ vs. other indications $(n=30$, $13.7 \%), p \leq 0.01$. In addition, the occurrence of postoperative complications differed according to the laparoscopy complexity level [I: $3(1.4 \%)$ vs. II: 128 (58.4\%) vs. III: $36(16.4 \%)$ vs. IV: $52(23.7 \%), p \leq 0.01]$, ASA status [I: 43 (19.6\%) vs. II: 127 (58\%) vs. III: 48 (21.9\%) vs. IV: 1 $(0.5 \%), p \leq 0.01]$, occurrence of intraoperative complications (62 (28.3\%) vs. $157(71.7 \%, p \leq 0.01)$, and performance of adhesiolysis (143 (65.3\%) vs. $76(34.7 \%, p=0.03)$.

Relative to those without postoperative complications, patients with such complications were significantly older [42 (range 9-95) vs. 47 (range 15-88) years], with longer surgeries (80 (range 2-492) vs. 132 (range 27-350) min], greater $\mathrm{CO}_{2}$ use [150 (range 7.4-2060) vs. 230 (range 7.7-1158) 1], larger hemoglobin drops [1.0 (range $-2.4-5.8$ ) vs. 1.3 (range - 4.2-8.5) g/dl], higher BMIs (25 (range 15.6-62.5) vs. 26.1 (range 16.3-57.5) $\left.\mathrm{kg} / \mathrm{m}^{2}\right]$, and longer hospital stays [3 (range 0-41) vs. 5 (range $0-75$ ) days; all $p \leq 0.01$ ] (Table 4).

\section{Risk factor analysis: multivariate analysis}

On multivariate analysis, independent risk factors for the occurrence of intraoperative complications were age [odds ratio (OR), 1.03; 95\% confidence interval (CI), 1.01-1.04], surgery duration (OR, 1.02; 95\% CI, 1.02-1.03), carbon dioxide use (OR, 0.99; 95\% CI, 0.99-1.00), and surgical indication (all $p \leq 0.01$ ). In relation to the reference group (endometriosis), the intraoperative complication risk was significantly greater among patients with benign adnexal findings (OR, 2.58; 95\% CI 1.02-6.49; $p=0.04)$ and malignant adnexal findings (OR, 4.68; 95\% CI 1.58-13.56; $p \leq 0.01$; Table 5). Independent risk factors for postoperative complications were the duration of surgery (OR, 1.01; 95\% CI 1.01-1.02; $p \leq 0.01$ ), carbon dioxide use (OR, 0.99; $95 \%$ CI $0.99-1.00 ; p \leq 0.01$ ), hemoglobin drop (OR, 1.41; 95\% CI 1.21-1.65; $p \leq 0.01)$, and ASA status ( $p=0.04$; Table 6).

\section{ROC analysis}

ROC analysis revealed that surgery duration $>99.5 \mathrm{~min}$ (AUC 0.8) and age $>38$ years (AUC 0.7) were appropriate thresholds for the intraoperative complication risk. For postoperative complications, the thresholds were surgery duration $>94.5 \mathrm{~min}$ (AUC 0.7) and hemoglobin drop > $2.05 \mathrm{~g} /$ dl (AUC 0.6).

\section{Discussion}

In this large retrospective study based on prospective clinical data, the intraoperative and postoperative complication rates of laparoscopic gynecological surgeries were $5.6 \%$ and $6.5 \%$, respectively. Patient age, duration of surgery, carbon dioxide use, and surgical indication were independent risk factors for intraoperative complications. These complications were more frequent in patients with benign and malignant adnexal findings than in those with endometriosis (reference group). Risk factors for postoperative complications were duration of surgery, carbon dioxide use, hemoglobin drop, and ASA status, with ASA grade III associated with a greater risk than ASA grade I. These low complication rates are similar to those obtained in other studies and confirm the safety of laparoscopic interventions. The standardization of surgical procedures and identification of risk collectives might help to further reduce complication rates.

A few previous major studies also focused on the incidence of complications in laparoscopic gynecological surgeries. Chapron et al. evaluated surgical complication rates of diagnostic and operative procedures (29,966 cases) and found a total rate of $0.46 \%$ [17]. Similarly, Härkki-Siren et al. evaluated the nationwide incidence of laparoscopic complications in a cohort of 70,607 patients and assessed a rate of $0.36 \%$ [18]. A higher incidence of complications was recorded in the analysis of Mac Cordick et al. with a rate of $2.9 \%$ out of 743 procedures and Leonard et al. with $3 \%$ in a cohort of 1033 patients [19, 20].

Two main explanations can be offered for the difference between these rates and those obtained in the present study.

First, in contrast to the previous studies, the Clavien-Dindo classification, which includes minor complications (e.g., need for analgesic or antibiotic treatment) and thus results in higher overall complication rates, was used in this study [11].

For instance, the postoperative complications considered by Chapron et al. would be classified as of Clavien-Dindo grades $\geq$ III; the exclusion of minor complications can be argued to have led to the underestimation of complication occurrence [17]. In a study from Radosa et al., in which the Clavien-Dindo classification was used, the total complication rate among 7438 laparoscopies was 13\% [8].

Another possible explanation for the "increase" in complication rates is the development of laparoscopic techniques over time and their expanded application to more advanced procedures, with concurrent evolution of systems 
Table 3 Surgical parameters in correlation with intraoperative complications

Surgical parameters in correlation with intraoperative complications $(n=3351)$

\begin{tabular}{|c|c|c|c|}
\hline & \multicolumn{2}{|c|}{ Intraoperative complications } & \multirow[t]{2}{*}{$p$-value } \\
\hline & Yes & No & \\
\hline & Total $(\%)$ & & \\
\hline Indication & & & $\leq 0.01$ \\
\hline Uterus myomatosus & $44(23.4 \%)$ & $779(24.6 \%)$ & \\
\hline Benign adnexal finding & $35(18.6 \%)$ & $865(27.3 \%)$ & \\
\hline Malignant adnexal finding & $31(16.5 \%)$ & $84(2.7 \%)$ & \\
\hline Endometrial cancer & $23(12.2 \%)$ & $128(4.0 \%)$ & \\
\hline Cervical cancer & $17(9.0 \%)$ & $114(3.6 \%)$ & \\
\hline Endometriosis & $16(8.5 \%)$ & $521(16.5 \%)$ & \\
\hline Others & $16(8.5 \%)$ & $478(15.1 \%)$ & \\
\hline Urogynaecological indication & $6(3.2 \%)$ & $156(4.9 \%)$ & \\
\hline $\mathrm{CIN}$ & $0(0.0 \%)$ & $38(1.2 \%)$ & \\
\hline Malignancy & & & $\leq 0.01$ \\
\hline Yes & $76(40.4 \%)$ & $345(10.9 \%)$ & \\
\hline No & $112(59.6 \%)$ & $2818(89.1 \%)$ & \\
\hline Barakat-classification & & & $\leq 0.01$ \\
\hline Grade II & $87(46.3 \%)$ & $2397(75.8 \%)$ & \\
\hline Grade IV & $69(36.7 \%)$ & $276(8.7 \%)$ & \\
\hline Grade III & $32(17.0 \%)$ & $429(13.6 \%)$ & \\
\hline Grade I & $0(0.0 \%)$ & $61(1.9 \%)$ & \\
\hline ASA-classification & & & $\leq 0.01$ \\
\hline Grade II & $109(58.0 \%)$ & $1971(62.3 \%)$ & \\
\hline Grade III & $51(27.1 \%)$ & $335(10.6 \%)$ & \\
\hline Grade I & $27(14.4 \%)$ & $853(27.0 \%)$ & \\
\hline Grade IV & $1(0.5 \%)$ & $4(0.1 \%)$ & \\
\hline Adhesiolysis & & & 0.39 \\
\hline Yes & $115(61.2 \%)$ & $1835(58.0 \%)$ & \\
\hline No & $73(38.8 \%)$ & $1328(42.0 \%)$ & \\
\hline Body mass index & & & $\leq 0.01$ \\
\hline Normalweight & $79(42.0 \%)$ & $1470(46.5 \%)$ & \\
\hline Overweight & $38(20.2 \%)$ & $878(27.8 \%)$ & \\
\hline Obesity I & $32(17.0 \%)$ & $414(13.1 \%)$ & \\
\hline Obesity III & $19(10.1 \%)$ & $112(3.5 \%)$ & \\
\hline Obesity II & $12(6.4 \%)$ & $186(5.9 \%)$ & \\
\hline \multirow[t]{3}{*}{ Underweight } & $8(4.3 \%)$ & $103(3.3 \%)$ & \\
\hline & \multicolumn{2}{|c|}{ Median (minimum-maximum) } & \\
\hline & Yes & No & \\
\hline Age (years) & $50(21-87)$ & $42(9-95)$ & $\leq 0.01$ \\
\hline Body mass index $\left(\mathrm{kg} / \mathrm{m}^{2}\right)$ & $26.1(16.3-59.5)$ & $25(15.6-62.5)$ & $\leq 0.01$ \\
\hline Operative time (min) & $180(24-492)$ & $79(2-415)$ & $\leq 0.01$ \\
\hline Carbon dioxide use (1) & $255(7.7-1373)$ & $153(7.4-2060)$ & 0.02 \\
\hline Hemoglobin drop (g/dl) & $1.5(-4.2-5.7)$ & $1,0(-2.2-8.5)$ & $\leq 0.01$ \\
\hline Length of hospital stay (days) & $7(0-75)$ & $3(0-72)$ & $\leq 0.01$ \\
\hline
\end{tabular}

CIN cervical intraepithelial neoplasia, ASA Classification American society of anaesthesiologists classification $(n=3351)$ 
Table 4 Surgical parameters in correlation with postoperative complications

Surgical parameters in correlation with postoperative complications $(n=3351)$

Postoperative complications

Yes

Total (\%)

Indication

Uterus myomatosus

Benign adnexal finding

Others

Endometriosis

Endometrial cancer

Malignant adnexal finding

Urogynecological indication

Cervical cancer

CIN

Malignancy

Yes

No

Barakat-classification

Grade II

Grade IV

Grade III

Grade I

ASA-classification

Grade II

Grade III

Grade I

Grade IV

Adhesiolysis

Yes

No

Body mass index

Normalweight

Overweight

Obesity I

Obesity III

Obesity II

Underweight

Intraoperative complications

Yes

No

Type of complication

Laparoconversion

Organ injury

Blood transfusion

Resuscitation

Skin emphysema

Bleeding
$60(27.4 \%)$

$35(16.0 \%)$

$30(13.7 \%)$

$24(11.0 \%)$

$23(10.5 \%)$

$20(9.1 \%)$

$15(6.8 \%)$

$10(4.6 \%)$

$2(0.9 \%)$

$58(26.5 \%)$

$161(73.5 \%)$

$128(58.4 \%)$

$52(23.7 \%)$

$36(16.4 \%)$

$3(1.4 \%)$

$127(58.0 \%)$

$48(21.9 \%)$

$43(19.6 \%)$

$1(0.5 \%)$

$143(65.3 \%)$

$76(34.7 \%)$

$90(41.1 \%)$

$61(27.9 \%)$

$32(14.6 \%)$

$17(7.8 \%)$

$13(5.9 \%)$

$6(2.7 \%)$

$62(28.3 \%)$

157 (71.7\%)

$40(18.3 \%)$

$13(5.9 \%)$

$7(3.2 \%)$

$1(0.5 \%)$

$1(0.5 \%)$

$0(0.0 \%)$
No

$p$-value

$$
\leq 0.01
$$

$763(24.4 \%)$

$865(27.6 \%)$

$464(14.8 \%)$

$513(16.4 \%)$

$128(4.1 \%)$

$95(3.0 \%)$

147 (4.7\%)

$121(3.9 \%)$

$36(1.1 \%)$

$\leq 0.01$

$363(11.6 \%)$

$2769(88.4 \%)$

$\leq 0.01$

$2356(75.2 \%)$

$293(9.4 \%)$

$425(13.6 \%)$

$58(1.9 \%)$

$\leq 0.01$

$1953(62.4 \%)$

$338(10.8 \%)$

$837(26.7 \%)$

$4(0.1 \%)$

0.03

$1807(57.7 \%)$

$1325(42.3 \%)$

0.06

$1459(46.6 \%)$

$855(27.3 \%)$

$414(13.2 \%)$

$114(3.6 \%)$

$185(5.9 \%)$

$105(3.4 \%)$

$126(4.0 \%)$

3006 (96.0\%)

$52(1.7 \%)$

$62(2.0 \%)$

$10(0.3 \%)$

$1(0.0 \%)$

$0(0.0 \%)$

$1(0.0 \%)$ 
Table 4 (continued)

\begin{tabular}{llll}
\hline & \multicolumn{2}{l}{ Median (minimum-maximum) } & \\
\cline { 2 - 3 } & Yes & No & $\leq 2(9-95)$ \\
\hline Age (years) & $47(15-88)$ & $25.0(15.6-62.5)$ & $\leq 0.01$ \\
\hline Body mass index $\left(\mathrm{kg} / \mathrm{m}^{2}\right)$ & $26.1(16.3-57.5)$ & $80(2-492)$ & $\leq 0.01$ \\
Operative time (min) & $132(27-350)$ & $150(7.4-2060)$ & $\leq 0.01$ \\
Carbon dioxide use (1) & $230(7.7-1158)$ & $1.0(-2.4-5.8)$ & $\leq 0.01$ \\
Hemoglobin drop (g/dl) & $1.3(-4.2-8.5)$ & $3(0-41)$ & $\leq 0.01$ \\
Length of hospital stay (days) & $5(0-75)$ & &
\end{tabular}

CIN cervical intraepithelial neoplasia, ASA Classification American Society of Anaesthesiologists Classification $(n=3351)$

Table 5 Multivariate analysis of factors associated with the occurrence of intraoperative complications

Multivariate analysis of factors associated with the occurrence of intraoperative complications $(n=3351)$

\begin{tabular}{lllr}
\hline & Odds Ratio & $95 \%$ CI & $p$-value \\
\hline Age (years) & 1.03 & $1.01-1.04$ & $\leq 0.01$ \\
Operative time (min) & 1.02 & $1.02-1.03$ & $\leq 0.01$ \\
Carbon dioxide use (1) & 0.99 & $0.99-1.00$ & $\leq 0.01$ \\
Indication (vs. endometriosis) & & & $\leq 0.01$ \\
Malignant adnexal finding & 4.68 & $1.58-13.56$ & $\leq 0.01$ \\
Benign adnexal finding & 2.58 & $1.02-6.49$ & 0.04 \\
Uterus myomatosus & 2.11 & $0.94-4.74$ & 0.07 \\
Cervical cancer & 0.83 & $0.29-2.31$ & 0.72 \\
Endometrial cancer & 0.94 & $0.32-2.69$ & 0.90 \\
Urogynaecological indication & 0.41 & $0.13-1.35$ & 0.14 \\
CIN & 0.00 & $0.00-1.00$ & 0.99 \\
Others & 1.88 & $0.69-5.09$ & 0.21 \\
\hline
\end{tabular}

CIN cervical intraepithelial neoplasia, $95 \%$ CI 95\% confidence interval, vs. versus $(n=3351)$

Table 6 Multivariate analysis of factors associated with the occurrence of postoperative complications

Multivariate analysis of factors associated with the occurrence of postoperative complications $(n=3351)$

\begin{tabular}{lllr}
\hline & Odds ratio & $95 \%$ CI & $p$-value \\
\hline Operative time (min) & 1.01 & $1.01-1.02$ & $\leq 0.01$ \\
Carbon dioxide use (1) & 0.99 & $0.99-1.00$ & $\leq 0.01$ \\
Hemoglobin drop (g/dl) & 1.41 & $1.21-1.65$ & $\leq 0.01$ \\
ASA-classification & & & 0.04 \\
$\quad$ I vs. II & 1.05 & $0.70-1.58$ & 0.81 \\
I vs. III & 1.76 & $1.05-2.95$ & 0.03 \\
I vs. IV & 6.57 & $0.65-66.63$ & 0.11 \\
\hline
\end{tabular}

ASA-Classification American Society of Anaesthesiologists Classification, 95\% CI 95\% confidence interval; vs. $=$ versus $(n=3351)$ used to classify laparoscopic surgeries. The classification of Chapron et al. was used in most previous studies to characterize the complexity of laparoscopic interventions (as diagnostic, minor, major, and advanced) [17].

The "advanced" category of this classification encompasses levels III and IV of the Barakat classification used in this study. Advanced procedures comprised $9.4-11.5 \%$ of surgeries in the previous studies, and comparable (Barakat levels III and IV) procedures comprised $24.1 \%$ of surgeries in the present study.

As in this study, Mirhashemi et al. found that the surgical indication was an independent risk factor for intraoperative complications; they reported lower rates associated with endometriosis and ovarian cystectomy [21]. Similarly, Saidi et al. reported that ovarian cystectomy was associated with the lowest rate of common complications [7]. We found that endometriosis was associated with a lower rate of intraoperative complications than were benign and especially malignant adnexal findings. Significant correlations between malignancy and complications have been reported previously $[9,22]$. The differences between these findings and ours may be explained by differences in study design; in the present study, we examined a large sample of patients undergoing a wide range of gynecological interventions.

Increasing age was an independent risk factor for the occurrence of intraoperative complications in this study. Three recent studies with designs similar to that of the present study yielded similar results. Age was an independent risk factor for complications of gynecological laparoscopic interventions in a sample of 1451 cases (along with previous radiation therapy and malignant diagnosis), and for bowel injury during hysterectomy in a cohort of 15,557 patients (along with endometriosis, and abdominal surgical approach) [9, 23]. Aside from surgery type, increasing age was the most important predictor of complications of laparoscopic gynecological surgery in a sample of 843 patients; the risk of operative injury or bleeding was five times greater among women aged $\geq 35$ years than among 
those aged $<35$ years [21]. In the present study, we found that intraoperative complications were more likely to occur in women aged $>38$ years. Older age may be a significant risk factor in this context due to the overall higher incidence of comorbidities with increasing age.

Of note, malignancy was not identified as a risk factor in our analysis, as it was in a previous study [9]. Chi et al.'s sample included more surgeries with malignant $(n=724)$ than with benign $(n=691)$ indications, whereas the majority of interventions examined in the present study were performed due to benign indications [9]. Thus, this discrepancy may be attributable to the larger percentage of malignant cases in Chi et al.'s smaller cohort [9].

The duration of surgery (i.e., longer operative time) was an independent risk factor for intraoperative and postoperative complications in this study. Two previous studies yielded similar results. In one study, the operative time (along with arterial hypertension, chronic obstructive pulmonary disease, and ASA status) was an independent risk factor for postoperative complications of 9145 laparoscopic procedures performed for the treatment of endometrial cancer [24]. In the other study, the operative time was associated independently with 30-day complications after laparoscopic and robotic hysterectomies [28]. Longer operative times can be associated with greater intraoperative blood loss and thus greater intraoperative and postoperative transfusion requirements and an increased risk of surgical site infection [25, 26]. They can also result in increased postoperative pain and increased risks of deep vein thrombosis and pulmonary artery embolism [27].

The ASA status describes patients' preoperative health and is a predictor of postoperative mortality and outcomes of laparoscopic and open surgeries [29]. In this study, ASA grade III was associated with a significantly greater risk of postoperative complications than was ASA grade I. Two previous studies yielded similar results. The ASA status was an independent risk factor for postoperative complications of laparoscopic procedures in a sample of 9145 patients with endometrial cancer, and higher ASA status was a significant predictor of postoperative complications and length of hospitalization $[24,30]$. Dean stated that this factor was useful for preoperative risk assessment [30].

The postoperative complication risk was also associated with the hemoglobin drop in this study. This variable has been reported as an independent risk factor for postoperative complications of other types of procedure (e.g., [31]). The hemoglobin drop presumably correlates directly with intraoperative blood loss and thus a greater postoperative transfusion requirement (a Clavien-Dindo grade II complication).

Carbon dioxide use was a risk factor for intraoperative and postoperative complications in this study. ORs for this variable were $<1$, indicating that risk increased with reduced carbon dioxide use. For intraoperative complications, this association may reflect the conversion of laparoscopy to laparotomy (with no carbon dioxide use). We could not find a report on a comparable assessment of the association of carbon dioxide use with complications in the literature.

Based on the findings of this study, we can identify a representative risk collective, who may benefit from close postoperative monitoring and surveillance. The implementation of a standardized approach to surgical management and procedures that reduces the surgery duration and involves preoperative hemoglobin optimization might contribute further to the prevention of complications. Patients with ASA statuses $>$ III might benefit from a multi-disciplinary approach to the preoperative optimization of health status.

\section{Limitations}

This study was conducted at a single center, which limits the generalizability of the findings. However, the inclusion of a large number of patients in the analysis helps to minimize this limitation. In addition, possible bias in the recording of surgical complications was avoided by having an advanced medical student (rather than a layperson) perform data acquisition using a standardized classification system [32]. In addition, although many studies have revealed a significant correlation between surgeon expertise and the occurrence of complications $[18,19]$, we did not consider this variable as a possible risk factor in our analysis. However, highly qualified and experienced surgeons perform all laparoscopic procedures at our institution.

\section{Conclusion}

In this large, single-center study, the overall complication rate for laparoscopic gynecological surgeries was low and independent risk factors for such complications were identified. Patients aged $>38$ years, surgery duration $>99$ min, benign or malignant adnex findings were at higher risk for intraoperative and patients with surgery duration $>94 \mathrm{~min}$, hemoglobin drop $>2 \mathrm{~g} / \mathrm{dl}$, ASA status III at higher risk for postoperative complications. The findings can be used to develop strategies aiming to further reduce intraoperative and postoperative complications of these procedures.

Acknowledgements This research project was registered in the German Register of Clinical Studies (no. DRKS00013790, 2018).

Author contribution ACK, MPR, JSMZ, LS, SF, AH, PS, ZT, GW, CGR, EFS and JCR designed the study and drafted the manuscript. ACK collected the data. In addition, each author has read and approved the final version of the manuscript. 
Funding Open Access funding enabled and organized by Projekt DEAL. This work has been funded by research grants from Saarland University Hospital (HOMFOR 2041601).

Availability of data and material The dataset used and analyzed during the current study is available from the corresponding author on reasonable request.

Code availability Not applicable.

\section{Declarations}

Conflict of interest The authors declare no conflict of interest regarding the submitted work. Regarding interactions outside the submitted work, Professor Dr. Julia Caroline Radosa has received travel grants from Medac GmbH (Wedel, Germany), Gedeon Richter (Budapest, Hungary), and Celgene (Summit, USA), Daiichi Sankyo (Tokio, Japan), Pfizer (New York City, USA) and was an honorary speaker for Pfizer (New York City, USA) and Clovis Oncology in the past. Dr. Lisa Stotz has received travel grants from Medac $\mathrm{GmbH}$ (Wedel, Germany) and Celgene (Summit, USA) outside the submitted work in the past. Dr. Julia Sarah Maria Zimmermann was an honorary speaker for Clovis Oncology in the past. Dr. Panagiotis Sklavounos was an honorary speaker for Pfizer, Astra Zeneca, Clovis Oncology und KLS Martin in the past. Professor Dr. Erich-Franz Solomayer is receiving grants from the University of Saarland, Storz, and Erbe; personal fees and other compensation from Roche (Basel, Switzerland), Pfizer (New York City, USA), Celgene (Summit USA), Amgen (Thousand Oaks, USA), and Astra Zeneca (Cambridge, GB); other fees from Johnson \& Johnson (New Brunswick, USA), Novartis (Basel, Switzerland), Tesaro (Waltham, USA), Medac GmbH (Wedel, Germany), MSD (Kenilworth, USA), Vifor (Sankt Gallen, Switzerland), Gedeon Richter (Budapest, Hungary), Takeda (Tokyo, Japan), and AGE (Buchholz, Germany) outside the submitted work.

Ethics approval This study was performed in line with the principles of the Declaration of Helsinki. Approval was granted by the Ethics Committee of University of Saarland (Date 16.02.2018/No. 19/18).

Consent to participate Informed consent was obtained from all patients included in the study.

Consent for publication The authors affirm that all patients provided consent for publication.

Open Access This article is licensed under a Creative Commons Attribution 4.0 International License, which permits use, sharing, adaptation, distribution and reproduction in any medium or format, as long as you give appropriate credit to the original author(s) and the source, provide a link to the Creative Commons licence, and indicate if changes were made. The images or other third party material in this article are included in the article's Creative Commons licence, unless indicated otherwise in a credit line to the material. If material is not included in the article's Creative Commons licence and your intended use is not permitted by statutory regulation or exceeds the permitted use, you will need to obtain permission directly from the copyright holder. To view a copy of this licence, visit http://creativecommons.org/licenses/by/4.0/.

\section{References}

1. Siddaiah-Subramanya M, Tiang K, Nyandowe M (2017) A new era of minimally invasive surgery: progress and development of major technical innovations in general surgery over the last decade. Surg J 03(04):e163-e166. https://doi.org/10.1055/s-00371608651

2. Scheib SA, Tanner E, Green IC, Fader AN (2014) Laparoscopy in the morbidly obese: physiologic considerations and surgical techniques to optimize success. J Minim Invas Gynecol 21(2):182195. https://doi.org/10.1016/j.jmig.2013.09.009

3. Kasai M et al (2018) Laparoscopic versus open major hepatectomy: a systematic review and meta-analysis of individual patient data. Surgery 163(5):985-995. https://doi.org/10.1016/j.surg. 2018.01.020

4. Medeiros LR, Stein AT, Fachel J, Garry R, Furness S (2008) Laparoscopy versus laparotomy for benign ovarian tumor: a systematic review and meta-analysis. Int J Gynecol Cancer 18(3):387-399. https://doi.org/10.1111/j.1525-1438.2007.01045.x

5. Zhang Y, Fan S, Xiang Y, Duan H, Sun L (2015) Comparison of the prognosis and recurrence of apparent early-stage ovarian tumors treated with laparoscopy and laparotomy: A meta-analysis of clinical studies. BMC Cancer. https://doi.org/10.1186/ s12885-015-1604-3

6. Galaal K, Donkers H, Bryant A, Lopes AD (2018) Laparoscopy versus laparotomy for the management of early stage endometrial cancer. Cochrane Database Syst Rev 2018(10):2. https://doi.org/ 10.1002/14651858.CD006655.pub3

7. Saidi MH, Vancaillie TG, White AJ, Sadler RK, Akright BD, Farhart SA (1996) Complications of major operative laparoscopy. A review of 452 cases. J Reprod Med 41(7):471-476

8. Radosa MP et al (2014) Standardised registration of surgical complications in laparoscopic-gynaecological therapeutic procedures using the Clavien-Dindo classification. Geburtshilfe Frauenheilkd 74(8):752-758. https://doi.org/10.1055/s-0034-1382925

9. Chi DS et al (2004) Ten-year experience with laparoscopy on a gynecologic oncology service: analysis of risk factors for complications and conversion to laparotomy. Am J Obstet Gynecol 191(4):1138-1145. https://doi.org/10.1016/j.ajog.2004.05.004

10. Irlbeck T, Zwißler B, Bauer A (2017) ASA classification: transition in the course of time and depiction in the literature. Anaesthesist. https://doi.org/10.1007/s00101-016-0246-4

11. Dindo D, Demartines N, Clavien PA (2004) Classification of surgical complications: a new proposal with evaluation in a cohort of 6336 patients and results of a survey. Ann Surg 240(2):205-213. https://doi.org/10.1097/01.sla.0000133083.54934.ae

12. Radosa $\mathbf{J}$ et al (2019) Impact of different intraoperative $<\mathrm{scp}>\mathrm{CO}</$ scp $>2$ pressure levels ( 8 and $15 \mathrm{mmHg}$ ) during laparoscopic hysterectomy performed due to benign uterine pathologies on postoperative pain and arterial $<\mathrm{scp}>\mathrm{pCO}</ \mathrm{scp}>2$ : a prospective randomised controlled clinical trial. BJOG An Int J Obstet Gynaecol 126(10):1276-1285. https://doi.org/10.1111/ 1471-0528.15826

13. Radosa JC et al (2016) Postoperative quality of life and sexual function in premenopausal women undergoing laparoscopic myomectomy for symptomatic fibroids: a prospective observational cohort study. doi: https://doi.org/10.1371/journal.pone. 0166659.

14. Radosa JC et al (2013) Five minutes of extended assisted ventilation with an open umbilical trocar valve significantly reduces postoperative abdominal and shoulder pain in patients undergoing laparoscopic hysterectomy. Eur J Obstet Gynecol Reprod Biol 171(1):122-127. https://doi.org/10.1016/j.ejogrb.2013.08.014

15. Radosa JC et al (2016) Influence of the preoperative decisionmaking process on the postoperative outcome after hysterectomy 
for benign uterine pathologies. Geburtshilfe Frauenheilkd 76(4):383-389. https://doi.org/10.1055/s-0041-110396

16. Radosa JC et al (2014) Influences of different hysterectomy techniques on patients' postoperative sexual function and quality of life. J Sex Med 11(9):2342-2350. https://doi.org/10.1111/jsm. 12623

17. Chapron C et al (1998) Surgical complications of diagnostic and operative gynaecological laparoscopy: a series of 29966 cases.

18. Härkki-Sirén P, Kurki T (1997) A nationwide analysis of laparoscopic complications. Obstet Gynecol 89(1):108-112. https://doi. org/10.1016/S0029-7844(96)00390-0

19. Mac Cordick C, Lécuru F, Rizk E, Robin F, Boucaya V, Taurelle $\mathrm{R}$ (1999) Morbidity in laparoscopic gynecological surgery: results of a prospective single-center study. Surg Endosc 13(1):57-61. https://doi.org/10.1007/s004649900898

20. Leonard F, Lecuru F, Rizk E, Chasset S, Robin F, Taurelle R (2000) Perioperative morbidity of gynecological laparoscopy. A prospective monocenter observational study. Acta Obstet Gynecol Scand 79(2):129-134. https://doi.org/10.1034/j.1600-0412.2000. 079002129.x

21. Mirhashemi R, Harlow BL, Ginsburg ES, Signorello LB, Berkowitz R, Feldman S (1998) Predicting risk of complications with gynecologic laparoscopic surgery. Obstet Gynecol 92(3):327-331. https://doi.org/10.1016/S0029-7844(98)00209-9

22. Sokol AI, Chuang K, Milad MP (2003) Risk factors for conversion to laparotomy during gynecologic laparoscopy. J Am Assoc Gynecol Laparosc 10(4):469-473. https://doi.org/10.1016/S10743804(05)60146-6

23. Zhu CR et al (2020) Risk factors for bowel injury in hysterectomy for benign indications. Obstet Gynecol 136(4):803-810. https:// doi.org/10.1097/AOG.0000000000004007

24. Singh S, Swarer K, Resnick K (2017) Longer operative time is associated with increased post-operative complications in patients undergoing minimally-invasive surgery for endometrial cancer. Gynecol Oncol 147(3):554-557. https://doi.org/10.1016/j.ygyno. 2017.09.024
25. Manara J et al (2020) Prolonged operative time increases risk of blood loss and transfusion requirements in revision hip surgery. Eur J Orthop Surg Traumatol. https://doi.org/10.1007/ s00590-020-02677-4

26. Cheng H, Chen BPH, Soleas IM, Ferko NC, Cameron CG, Hinoul $P$ (2017) Prolonged operative duration increases risk of surgical site infections: a systematic review. Surg Infect 18(6):722-735. https://doi.org/10.1089/sur.2017.089

27. Jason Abel E et al (2014) Surgical operative time increases the risk of deep venous thrombosis and pulmonary embolism in robotic prostatectomy. J Soc Laparoendosc Surg 18(2):282-287. https:// doi.org/10.4293/jsls.2014.00101

28. Catanzarite T, Saha S, Pilecki MA, Kim JYS, Milad MP (2015) Longer operative time during benign laparoscopic and robotic hysterectomy is associated with increased 30-day perioperative complications. J Minim Invasive Gynecol 22(6):1049-1058. https://doi.org/10.1016/j.jmig.2015.05.022

29. Daabiss $M$ (2011) American society of anaesthesiologists physical status classification. Indian J Anaesthesia 55(2):111-115. https:// doi.org/10.4103/0019-5049.79879

30. Dean M (2001) Predictors of complications and hospital stay in gynecologic cancer surgery. Obstet Gynecol 97(5):721-724. https://doi.org/10.1016/s0029-7844(00)01198-4

31. Jung DH et al (2013) Impact of perioperative hemoglobin levels on postoperative outcomes in gastric cancer surgery. Gastric Cancer 16(3):377-382. https://doi.org/10.1007/s10120-012-0196-8

32. Dindo D, Hahnloser D, Clavien PA (2010) Quality assessment in surgery: riding a lame horse. Ann Surg 251(4):766-771. https:// doi.org/10.1097/SLA.0b013e3181d0d211

Publisher's Note Springer Nature remains neutral with regard to jurisdictional claims in published maps and institutional affiliations. 